\title{
Pediatric endocrine society survey of diabetes practices in the United States: What is the current state?
}

\author{
| Guttmann-Bauman ${ }^{1}$ ( ) | P Thornton ${ }^{2}$ | S Adhikari ${ }^{3}$ | K Reifschneider ${ }^{4}$ | MA Wood ${ }^{5}$ | \\ T Hamby ${ }^{6}$ | K Rubin ${ }^{7}$
}

${ }^{1}$ Department of Pediatrics, Oregon Health and Science University (OHSU), Portland, Oregon

${ }^{2}$ Cook Children's Medical Center, Fort Worth, Texas

${ }^{3}$ UT Medical Center, Children's Medical Center, Dallas, Texas

${ }^{4}$ Eastern Virginia Medical School - Children's Hospital of the Kings Daughters, Norfolk, Virginia

${ }^{5}$ University of Michigan Medical School, Ann Arbor, Michigan

${ }^{6}$ Department of Research Operations, Cook Children's Health Care System, Fort Worth, Texas

${ }^{7}$ University of Connecticut School of Medicine, Department of Pediatrics and Head of Clinical Care Innovation, Connecticut Children's Medical Center, Hartford,

Connecticut

\section{Correspondence}

Ines Guttmann-Bauman, MD, Oregon Health and Science University (OHSU), 707 SW

Gaines St, Portland, OR 97239.

Email: guttmann@ohsu.edu

\begin{abstract}
Background: The Practice Management Committee (PMC) of the Pediatric Endocrine Society (PES) conducted a survey of its membership in February/March, 2016 to assess the current state of pediatric diabetes care delivery across multiple practice types in the United States.

Methods: The PES distributed an anonymous electronic survey (Survey Monkey) via email to its membership and requested that only one survey be completed for each practice.

Results: Ninety-three unique entries from the US were entered into analysis. Care is predominantly delivered by multidisciplinary teams, based at academic institutions (65.6\%), with $>85 \%$ of the provider types being physicians. Each 1.0 full time equivalent certified diabetes educators serves on average 367 diabetic youth. Fee-for-service remains the standard method of reimbursement with $57 \%$ of practices reporting financial loss. Survey respondents identified under-reimbursement as a major barrier to improving patient outcomes and lack of behavioral health $(\mathrm{BH})$ providers as a key gap in services provided.

Conclusions: Our survey reveals wide variation in all aspects of pediatric diabetes care delivery in the United States. Pediatric Endocrinologists responding to the survey identified a lack of resources and the current fee for service payment model as a major impediment to practice and the lack of integrated $\mathrm{BH}$ staff as a key gap in service. The respondents strongly support its organizations' involvement in the dissemination of standards for care delivery and advocacy for a national payment model aligned with chronic diabetes care in the context of our emerging value-based healthcare system.
\end{abstract}

\section{KEYWORDS}

care delivery, diabetes practice, population health

\section{1 | INTRODUCTION}

Type 1 diabetes, one of the most common chronic disorders of childhood, is increasing in incidence, ${ }^{1}$ affecting 1 in 300 children in the United States by the age of $18 .^{2}$ Type 2 diabetes, a condition virtually unknown in children until the 1990s, is rising in prevalence as well, requiring additional resources within pediatric endocrine practices. ${ }^{3}$

According to the International Society for Pediatric and Adolescent Diabetes (ISPAD) 2014 Clinical Practice Consensus Guidelines Compendium, the ultimate goal in diabetes care delivery is to "provide care that results in normal growth and development, high quality of life, and lowest possible risk of acute and long-term complications. This is best accomplished by helping children and families become proficient in self-management, remain motivated throughout childhood and adolescence while mentoring children to develop into independent, healthy adults". 4,5

Despite significant advances in diabetes therapeutic options and technology in the past 2 decades, optimal care delivery models that empower patients to benefit from these advances have not been well-described or standardized nationwide. One well-recognized challenge to optimizing diabetes care delivery in the United States is the progressive decline in traditional fee for service (FFS) payment, 
resulting in a greater need for institutional support. This decline has impeded pediatric endocrinologists' ability to financially implement their care teams and to maintain and evaluate optimal care models. Under-resourced diabetes practices result in inadequate staffing and lack of key professional team members whose role is to engage in education and care coordination, while addressing social stressors and behavioral health $(\mathrm{BH})$ concerns.

Optimizing diabetes care requires that we expand our understanding of current care delivery models, their strengths and weaknesses, and the barriers to meeting patients' needs. To address this, the Practice Management Committee (PMC) of the Pediatric Endocrine Society (PES) surveyed the membership to determine clinical and financial information, including the average number of patients with either type 1 or type 2 diabetes served in their practices, the type of staff providing care, and how they are reimbursed. Most of the questions focused on care delivery for patients with type 1 diabetes, as they constitute the majority in most diabetes practices. The membership was also asked if they felt their current staffing levels and reimbursement enabled them to achieve the ISPAD goals. In addition, the survey authors were interested in knowing to what extent survey responders desire PES to engage on their behalf in advocacy efforts toward healthcare policy makers and payers.

\section{2 | METHODS}

An anonymous, self-report survey of pediatric diabetes practices was formulated on Survey Monkey by members of the PMC and distributed by PES to its members from February to March, 2016. Although sent to all members, survey instructions requested that the senior member (division chief or head of diabetes section) complete the survey as both the scope and complexity of the survey ( $>70$ complex questions) warranted practice-wide vs individual provider participation. The project was reviewed by the Research Advisory Council of the PES, who determined it was a quality improvement rather than a research initiative and did not require IRB approval. Survey questions were grouped into 4 sections addressing the following topics: (1) practice settings and staffing characteristics; (2) behavioral/mental health care; (3) pediatric endocrinologists' satisfaction with and concerns about current state of practice; and (4) patient outcome data. Data requested in this last section was similar to data requested by US News and World Report for its Best Children's Hospital rankings, and thus the data was reported only for those institutions who participated in this survey. Participants were allowed to report the data using the databases of their choice, and we were not able to independently verify the accuracy of the submitted answers. Respondents were permitted to skip questions, so we reported the number of respondents for each item. Responses were transferred to SAS Enterprise version 6.1 for further statistical analyses. Non-structured information was categorized into themes and reported as such.

\section{3 | RESULTS}

Surveys were sent out to all the membership of the PES. There were 101 responses from the US. Thirteen responses (12.9\%) were from the same practices with identical internet protocol addresses: 5 were double entries, and 1 was a triple entry. The final dataset included 93 unique entries. Because respondents were not required to respond to each item, there were substantial missing values; hence, the number of responding participants is stated for each item. In total, data regarding the care of 73170 pediatric patients with type 1 diabetes and 10440 patients with type 2 diabetes were reported. There is currently no reliable database that would specify the number of Pediatric Endocrinology practices in the US, but we estimate that our survey covered practices that take care of approximately $30 \%$ to $40 \%$ of all youth with diabetes in the US. ${ }^{1}$

\section{4 | PRACTICE TYPE AND KEY CHARACTERISTICS}

The majority of responders were academic medical centers (65\%), accounting for a total of 66120 patients (Table 1). The majority of respondents (85\%) were hospital based, with few (12\%) private practice respondents. Primary providers in most practices were physicians $-86.0 \%$ of 57 academic centers, $83.3 \%$ of 18 non-academic centers, and $100 \%$ of 10 private practices. Advanced practice providers (Nurse Practitioners and Physician Assistants) accounted for a much smaller fraction of primary providers.

Characteristics of responding academic, non-academic and private practices are displayed in Table 1 (practice size) and Table 2 (patient load/staff ratio).

\section{5 | NEW-ONSET CARE}

The average ( \pm SD) number of newly diagnosed type 1 and 2 diabetes patients per year was $97.4 \pm 84.2(n=89)$ and $22.3 \pm 25.4(n=82)$, respectively.

Initial education of newly diagnosed patients with T1D was predominantly provided in the inpatient setting in $71.0 \%$ of 89 responding centers. The duration of new patient education was difficult to determine because respondents described a wide variety of practices, often reporting combining inpatient- and outpatient-administered modules. Diabetes education was provided by certified diabetes educator (CDE) employed by the clinics in $72 \%$ of responding institutions and by hospital inpatient nursing staff alone in $11.8 \%$. The remaining $16.2 \%$ of practices reported using different types of resources. Group sessions were used by less than half respondents (48\%) during their education process, either at onset or shortly after the onset of type 1 diabetes. Of 91 respondents, 55.0\% reported that their practices did not have a specific, separate education process for the initial education of type 2 diabetes patients.

\section{6 | ESTABLISHED AMBULATORY CARE}

Patients time slots scheduled with physicians varied, with 30 minutes per patient the most common (60\%), while 15 to 20 minutes slots were reported by $22.6 \%$ of physicians and $>30$ minutes by $17.2 \%$. 
TABLE 1 Descriptive statistics for numbers of type 1 and type 2 diabetes patients by region and provider type

\begin{tabular}{|c|c|c|c|c|c|c|c|c|c|c|}
\hline \multirow[b]{2}{*}{ Region } & \multirow[b]{2}{*}{ Diabetes } & \multicolumn{3}{|c|}{ Total } & \multicolumn{2}{|c|}{ Academic } & \multicolumn{2}{|c|}{ Non-academic } & \multicolumn{2}{|c|}{ Private } \\
\hline & & $n$ & $M$ & SD & $\bar{n}$ & $M$ & $n$ & $M$ & $n$ & $M$ \\
\hline \multirow[t]{2}{*}{ Total } & Total & 90 & 929 & 723 & 58 & 1140 & 19 & 664 & 11 & 374 \\
\hline & Type 2 & & 116 & 112 & & 138 & & 92 & & 49 \\
\hline \multirow[t]{2}{*}{ Midwest } & Total & 19 & 1126 & 729 & 13 & 1398 & 4 & 677 & 2 & 255 \\
\hline & Type 1 & & 1027 & 675 & & 1267 & & 641 & & 243 \\
\hline \multirow[t]{3}{*}{ Northeast } & Total & 22 & 712 & 446 & 17 & 733 & 3 & 780 & 2 & 423 \\
\hline & Type 1 & & 611 & 390 & & 644 & & 564 & & 400 \\
\hline & Type 2 & & 100 & 114 & & 89 & & 215 & & 23 \\
\hline \multirow[t]{2}{*}{ South } & Total & 29 & 931 & 677 & 18 & 1036 & 7 & 887 & 3 & 497 \\
\hline & Type 1 & & 770 & 585 & & 847 & & 780 & & 367 \\
\hline West & Type 2 & & 86 & 108 & & 140 & & 43 & & 20 \\
\hline
\end{tabular}

Advanced care providers had more time allotted for appointments; among the 64 respondents, $42.2 \%$ reported having 30 minutes and $46.9 \%$ reported having 45 minutes or longer. Survey responders found it difficult to quantify visit time with CDEs, who often do not schedule free standing appointments, but rather see patients together with other providers.

The average $( \pm S D)$ time that a patient with diabetes spends in a follow-up appointment, from check-in to check-out, was estimated to be $67.7 \pm 27.8$ minutes in the main clinic by 89 respondents and $57.2 \pm 20.8$ minutes in satellite clinics by 54 respondents. Seventyeight percent of 91 responding practices reported utilizing care coordination, and $70.7 \%$ of 65 practices reported having specific interventions for patients with socio-economic status challenges/ poor adherence and services to aid with transition to adult care. Very few practices (18.9\% of 90 reporting) reported utilizing videoconference telemedicine visits, and $35.9 \%$ of 89 practices reported having a patient/family advisory board.

\section{7 | ADHERENCE TO STANDARDS OF CARE}

Of the 47 reporting centers, practices regarding adherence to standards of care showed that $56.4 \%$ of type 1 diabetes patients had 4 or more visits per year, as recommended by ADA, and $86.8 \%$ of primary diabetes patients (including both type 1 and 2) had a face-to-face visit with a $C D E$ in the preceding year. Of the 46 reporting practices,
71.3\% and $46.4 \%$ of patients had face-to-face visits with a dietitian and a mental health specialist, respectively, in the preceding year. Median A1c value in patients with commercial insurance was $8.3 \pm 0.22 \%(68 \mathrm{nmol} / \mathrm{mol})$ in academic medical centers $(n=25)$, $8.41 \pm 0.27 \%(68 \mathrm{nmol} / \mathrm{mol})$ in non-academic centers $(n=8)$ and $7.75 \pm 0.4 \%(61 \mathrm{nmol} / \mathrm{mol})$ in private practices $(n=4)$. In patients with Medicaid, those values were $8.80 \pm 0.45 \%(73 \mathrm{nmol} / \mathrm{mol})$ in academic medical centers $(n=27), 8.98 \pm 0.47 \%(75 \mathrm{nmol} / \mathrm{mol})$ in non-academic centers $(n=8)$ and $8.18 \pm 0.72 \%(66 \mathrm{nmol} / \mathrm{mol})$ in private practices $(n=4)$. Among 48 reporting practices, $93.1 \%$ of eligible type 1 diabetes patients had thyroid tests performed in the past 2 years. Of type 1 and 2 diabetes patients $>10$ years, $89.7 \%$ had lipid screen performed in the past 5 years; among those who additionally had a duration of diabetes $>5$ years, $85.1 \%$ had urine microalbumin screen in the past year and $58.7 \%$ had retinal exam done in the preceding year. Patients of private practices $(n=8)$ tended to be more adherent to standards of screening; however, the number of private practices respondents was too small to draw conclusions about importance of this finding.

\section{8 | REIMBURSEMENT}

Fee-for-service was the main method of reimbursement as was reported by $82.0 \%$ of 89 responding practices. Table 3 illustrates the billing practices for support services.

TABLE 2 Number of patients with T1D per provider type

\begin{tabular}{|c|c|c|c|c|c|c|c|c|c|c|c|c|}
\hline & \multicolumn{3}{|c|}{ Total } & \multicolumn{3}{|c|}{ Academic } & \multicolumn{3}{|c|}{ Non-academic } & \multicolumn{3}{|c|}{ Private } \\
\hline & $n$ & $M$ & SD & $n$ & $M$ & SD & $n$ & $M$ & SD & $n$ & $M$ & SD \\
\hline \multicolumn{13}{|c|}{ Type 1 patients per... } \\
\hline Social worker & 61 & 2032 & 2831 & 48 & 2246 & 3150 & 12 & 1255 & 614 & 1 & 1083 & \\
\hline Dietitian & 75 & 750 & 545 & 54 & 824 & 581 & 16 & 514 & 344 & 4 & 761 & 587 \\
\hline Psychologist & 38 & 3455 & 3549 & 33 & 3601 & 3540 & 3 & 3827 & 4805 & 1 & 500 & \\
\hline Educators & 83 & 367 & 385 & 55 & 395 & 447 & 19 & 274 & 217 & 7 & 453 & 166 \\
\hline
\end{tabular}


TABLE 3 Billing and payment for services

\begin{tabular}{|c|c|c|c|c|c|c|}
\hline \multirow[b]{2}{*}{ Service } & \multicolumn{3}{|c|}{$\begin{array}{l}\text { Which of these } \\
\text { services are billed } \\
\text { for in your } \\
\text { practice? }\end{array}$} & \multicolumn{3}{|c|}{$\begin{array}{l}\text { If billing, are they } \\
\text { collecting } \\
\text { reimbursement? }\end{array}$} \\
\hline & n & $\begin{array}{l}\% \\
\text { Yes }\end{array}$ & $\begin{array}{l}\% \\
\text { No }\end{array}$ & n & $\%$ Yes & $\begin{array}{l}\% \\
\text { No }\end{array}$ \\
\hline \multicolumn{7}{|l|}{ CDE } \\
\hline One-on-one & 88 & $63 \%$ & $38 \%$ & 46 & $91 \%$ & $9 \%$ \\
\hline Group classes & 84 & $40 \%$ & $60 \%$ & 25 & $96 \%$ & $4 \%$ \\
\hline \multicolumn{7}{|c|}{ Clinical (behavior) therapy } \\
\hline $\begin{array}{l}\text { One-on-one } \\
\text { encounter }\end{array}$ & 82 & $43 \%$ & $57 \%$ & 29 & $90 \%$ & $10 \%$ \\
\hline Drop-by services & 79 & $11 \%$ & $89 \%$ & 7 & $100 \%$ & $0 \%$ \\
\hline \multicolumn{7}{|l|}{ Services } \\
\hline Social work & 83 & $12 \%$ & $88 \%$ & 5 & $80 \%$ & $20 \%$ \\
\hline Nutrition & 85 & $71 \%$ & $29 \%$ & 53 & $92 \%$ & $8 \%$ \\
\hline
\end{tabular}

Eighty-nine percent of 84 respondents reported that their practices received no reimbursement for non-face-to-face services. Of 86 practices, $15.1 \%$ reported financial profit from providing diabetes services, $57.0 \%$ reported loss and $27.9 \%$ reported not having access to the information. Of 13 profit-reporting practices, $53.8 \%$ were private practices, $15.4 \%$ were academic and $23.1 \%$ were non-academic practices. Thus the rate of taking care of patients with diabetes for a loss is a staggering $96.6 \%$ in a academic centers and $84.3 \%$ in nonacademic, hospital based practices.

\section{9 | BH SERVICES}

Of 87 respondents, 56.3\% reported having one or more $\mathrm{BH}$ specialists integrated in their team, $26.4 \%$ reported having access to those services within their institution and $17.2 \%$ reported no access to $\mathrm{BH}$ services. Of 81 respondents, $40.7 \%$ indicated that $\mathrm{BH}$ specialists in their practice see all new patients, and $43.5 \%$ of 85 respondents reported screening type 1 diabetes patients for mental health problems using a validated screening tool. Four barriers to mental health services were reported to be either significant or very significant barriers by most respondents: lack of insurance coverage by $76.5 \%$ of 81 respondents, lack of $\mathrm{BH}$ providers in the area by $61.4 \%$ of 83 respondents, lack of $\mathrm{BH}$ providers trained in diabetes in $69.5 \%$ of 82 respondents, and lack of institutional support for multidisciplinary approach by $59.3 \%$ of 81 respondents.

Eighteen $(23.7 \%)$ of 76 practices reported having $\mathrm{BH}$ specialists routinely see all clinic patients for prevention and guidance, with the rest reporting that those services are offered to psychologically challenged patients only. Of 65 participants reporting how $\mathrm{BH}$ services were reimbursed, $55.4 \%$ indicated the clinic covering the costs, $58.5 \%$ indicated FFS, 6.2\% indicated grant, and 6.2\% indicated foundation support.

\section{0 | PROVIDER SATISFACTION AND CONCERNS}

Seventy-six (89.4\%) of 85 responders indicated that they were very or somewhat satisfied with the duration and quality of care provided to their patients with type 1 diabetes at diagnosis. Among those 9 respondents who reported not being satisfied, $88.9 \%$ indicated that they would prefer more education to be available to the patients after the initial diagnosis on the outpatient basis and $87.5 \%$ of 8 responders would like to see a more consistent process of education, provided by specialized providers, with CDE qualification. Sixtyone $(71.7 \%)$ of 85 responders were satisfied with the time allotted for follow-up clinic visits and the current composition of their team. Table 4 illustrates major areas of concern. When asked for improvement suggestions, the greatest needs were expressed for $\mathrm{BH}$ support and higher reimbursement, so the staff can spend the time they need with the patient.

Regarding the reimbursement climate, $59.8 \%$ of 82 responders indicated that they are either very or somewhat dissatisfied with the current FFS model.

Last, $78.8 \%$ of 85 respondents thought it was somewhat or very important to create staffing guidelines for care of children with type 1 diabetes, that would be recognized as best practice and $>90 \%$ of 85 respondents indicated that PES should take the lead in establishing the standards.

\section{1 | DISCUSSION}

Our survey captured quantitative and qualitative data from all forms of clinical practices caring for over 80000 children with diabetes. Based on the SEARCH study data from 2010, 179387 US youth were estimated to have the diagnosis type 1 diabetes and 22820 of the type 2 diabetes. ${ }^{1}$ We found that pediatric diabetes care facilities in the US are concentrated in academic health centers and hospital based practices. It is not clear if the lack of private practice centers reporting is due to the fact that many do not care for patients with diabetes or that they did not respond to the survey. Physicians constitute the majority of primary providers across all practice settings with advanced practice providers significantly less frequently employed in that role. Consistent with their central role in diabetes care and education, we found that CDEs are the most common nonphysician diabetes team professional employed, with an approximate ratio of 1 full-time educator per 367 children in the practice. Registered dietitians (RDs) are the next most commonly employed nonMD professional, with an approximate ratio of $1 \mathrm{RD}$ for every $2 \mathrm{CDEs}$, although it was not possible to determine to what extent the RDs were utilized in a combined $\mathrm{CDE} / \mathrm{RD}$ role. $\mathrm{BH}$ providers are less commonly employed within the care team. Although no correlations were observed between staffing ratios and $\mathrm{A} 1 \mathrm{c}$, we recommend caution in accepting this negative finding, due to the insufficient number of practices reporting $\mathrm{A} 1 \mathrm{c}$ values to our survey. Future prospective studies are needed to ascertain which provider types and interventions result in the best outcomes. Registries, similar to one used for patients with cystic fibrosis would allow for more consistent data collection and a more accurate analysis.

Our A1c dataset is consistent with that of the T1D Exchange (5) and demonstrates that over $50 \%$ of children with diabetes do not achieve the ADA published A1c target of $<7.5 \%$. In addition, as has 
TABLE 4 Likert ratings for staffing and payment questions

\begin{tabular}{|c|c|c|c|c|c|c|}
\hline \multirow[b]{2}{*}{ Question } & \multirow[b]{2}{*}{$n$} & \multicolumn{5}{|l|}{$\%$} \\
\hline & & 1 & 2 & 3 & 4 & 5 \\
\hline \multicolumn{7}{|c|}{ Rate your agreement with the following Statements ${ }^{a}$} \\
\hline Need more MD providers & 83 & $8 \%$ & $20 \%$ & $28 \%$ & $13 \%$ & $30 \%$ \\
\hline $\begin{array}{l}\text { Need more non-MD providers } \\
\text { (APN/PA) }\end{array}$ & 80 & $14 \%$ & $14 \%$ & $36 \%$ & $18 \%$ & $19 \%$ \\
\hline Need more RN/CDE support & 84 & $4 \%$ & $8 \%$ & $15 \%$ & $27 \%$ & $45 \%$ \\
\hline Need more dietitian support & 83 & $4 \%$ & $5 \%$ & $23 \%$ & $35 \%$ & $34 \%$ \\
\hline $\begin{array}{l}\text { Need more mental health } \\
\text { support }\end{array}$ & 84 & $1 \%$ & $1 \%$ & $10 \%$ & $25 \%$ & $63 \%$ \\
\hline Need more reimbursement & 83 & $5 \%$ & $0 \%$ & $8 \%$ & $24 \%$ & $63 \%$ \\
\hline \multicolumn{7}{|c|}{ Rate the importance of each of the following ${ }^{b}$} \\
\hline $\begin{array}{l}\text { Increase fee via value-added } \\
\text { payment system }\end{array}$ & 77 & $1 \%$ & $1 \%$ & $17 \%$ & $35 \%$ & $45 \%$ \\
\hline $\begin{array}{l}\text { Availability of more coding } \\
\text { options }\end{array}$ & 77 & $8 \%$ & $5 \%$ & $25 \%$ & $32 \%$ & $30 \%$ \\
\hline $\begin{array}{l}\text { Reimbursement for non-face-to- } \\
\text { face services }\end{array}$ & 79 & $0 \%$ & $0 \%$ & $3 \%$ & $19 \%$ & $78 \%$ \\
\hline Bundled payments/capitation & 73 & $5 \%$ & $4 \%$ & $36 \%$ & $32 \%$ & $23 \%$ \\
\hline
\end{tabular}

a 1 , strongly disagree; 2 , disagree; 3 , neutral; 4 , agree; 5 , strongly agree.

b 1 , very unimportant; 2 , somewhat unimportant; 3 , neither important nor unimportant; 4, somewhat important; 5 , very important.

been reported for other pediatric chronic conditions, Medicaid-insured patients experience worse outcomes, irrespective of practice type.

A 30 minute visit time with the team professional was deemed to be adequate by a majority of providers, but the length of visit might be more dependent to the institutional constraints, rather than physicians' preference, so this information should be interpreted with caution. Patient-reported outcomes (PROs) were not captured by our survey.

Only $56 \%$ of patients met the standard of care for quarterly visits, despite the fact that missed visits are associated with poor glycemic control and preventable episodes of diabetic ketoacidosis. ${ }^{6}$ This finding underscores the need to explore the causes for missed visits and to guide potential interventions such as telehealth visits, flexible hours, and employing $\mathrm{BH}$ and social work (SW) as well as care coordinators to ensure that high-risk patients keep their appointments.

Meeting the needs of the most vulnerable patients and families requires access to care coordinators trained on linking patients and families to available community-based and $\mathrm{BH}$ services. Typically it is the role of SW, however only $56.3 \%$ of practices (primarily academic institutions) have those team members available.

Similarly, the implementation of a staged transition to adult care is not standardized, nor is the adoption of a specific new-onset process for type 2 diabetes patients whose medical and behavioral-social needs differ from the typical type 1 diabetes patient.

It is our opinion that the observed variability in staffing levels and other aspects of care delivery likely reflects leadership decisions, patient demographics, local culture, and financial resources. It is possible that this variability could be reduced if there was a national consensus statement establishing best practices for team composition, staffing ratios and core components of care delivery. We attribute the fact that $89 \%$ of the responders express satisfaction with the quality of care provided, while $90 \%$ recognize the need for additional
BH support, to "cognitive dissonance" related to a "learned helplessness" among under-resourced pediatric endocrinologists within their organizations. ${ }^{7}$

Slightly more than half of surveyed practices had a $\mathrm{BH}$ specialist integrated within their team, and only $23.7 \%$ reported those specialists providing preventive care and guidance within the diabetes clinic, indicating an understaffed and largely "reactive" model of BH intervention. The prevalence of psychological issues in diabetic youth is significantly higher than those without diabetes and is associated with decreased self-care, higher A1C, poor diabetes outcomes, and increased health costs. ${ }^{8,9}$ Psychosocial screening is recommended as a standard part of initial and ongoing care to identify families at risk for early emerging complications and non-adherence. ${ }^{10}$ Systematic reviews and meta-analyses found that the most effective interventions were those delivered to the family or groups of families, leading to improved health outcomes and lower cost of care. ${ }^{11-13}$ Despite this evidence, cost and low reimbursement are expressed by most respondents as the main barriers to integrating sufficient $\mathrm{BH}$ services within diabetes care teams (Table 4).

Survey results indicate that $71 \%$ of diabetes care teams admit their new-onset patients for education, despite the fact that ambulatory new-onset care for metabolically stable patients is equally effective and dramatically cheaper. ${ }^{14,15}$ The reality is that high-cost inpatient new-onset care will predominate until new payment models are in place that rewards healthcare entities to transition new-onset care to the outpatient setting. ${ }^{16,17}$

Although FFS represents a substantial portion of care delivered by diabetes teams nationwide, it does not cover laborious non-faceto-face care ${ }^{18}$. At least $57 \%$ of practices report negative margins on providing diabetes care which validates previous analyses by The Diabetes Working Group, ${ }^{19}$ and Melzer et al. ${ }^{20}$ We speculate that this is due to the low rate of billing for diabetes education, clinical behavioral therapy and social work services in the current system (Table 3) and by the huge amount of uncompensated time spent by CDEs on phone triage, insurance paperwork and education.

Most of the survey responders consider change in reimbursement model a high priority (Table 4). Although local innovation has established a proof-of-concept for a longitudinal bundled payment model for youth with T1D in 1 pediatric institution, it became unsustainable in the face of current health reform trends that are shifting more of the cost burden to families. ${ }^{21}$ As we adopt population health strategies and demonstrate the value diabetes care teams provide, we will be able to join the growing ranks of provider organizations that are implementing and evaluating value-based care and payment models. Until then, optimization of FFS reimbursement for RDs, CDEs, SWs and psychologists can partially offset the costs of care for youth with diabetes.

At the same time we aim for financial sustainability, we need to find ways to deliver quality care at lower costs. The introduction of new efficiencies and technology into care delivery will become increasingly important. Telemedicine is a rapidly expanding mode of care delivery, shown to increase patient engagement and expand touch points with their care team. A recent study by Wood et al demonstrated an improvement in ADA standard adherence with telemedicine visits. ${ }^{21}$ Low (19\%) adoption of telemedicine, indicated 
by our survey respondents represents another strategy to pursue, as more and more states and payers reimburse direct to patient remote visits. Based on our survey data and current healthcare trends, future areas of focus might also include how best to integrate the patient and family perspective into the care team, the development and validation of PROs, and the analysis of the key aspects of care delivery, technology, and payment models that optimize key diabetes outcomes across diverse healthcare settings. ${ }^{22}$

Succeeding in our rapidly changing and disruptive healthcare environment calls upon organizations and advocacy groups to partner together. PES recently formed a Special Interest Group for pediatric diabetes to represent the views of its membership, advocate on their behalf, and serve as a PES representative in relevant joint initiatives. Similarly, the T1D Exchange and the Juvenile Diabetes Research Foundation have partnered together to develop and disseminate a set of standardized diabetes outcome measures beyond A1c, to be used as common language to assess outcomes and conduct research. $^{23}$

In summary, our survey results provides information not recently or previously gathered on 93 US diabetes practices and demonstrates a wide variability in care delivery and team composition across practices. The greatest perceived barrier among respondents to improving care is the lack of financial resources and the greatest care gap is $\mathrm{BH}$.

A major limitation to interpreting the results of our survey is that responders were allowed to respond to questions of their choosing, and that the data reported could not be independently verified. This certainly brings up a concern for observational bias, which we acknowledge. Despite these limitations, the consistent themes that emerged resonate with the collective experience of front-line practicing pediatric endocrinologists and lead the authors to conclude that the survey findings represent the status of pediatric diabetes care teams in the US.

\section{ACKNOWLEDGEMENTS}

We thank PES for acknowledging the importance of diabetes care to its members, and for increasing its diabetes infrastructure. These survey findings underscore the need for PES and its "sister" organizations, like ISPAD and ADA to work together and transform pediatric diabetes care across the care continuum.

\section{Author contributions}

Ines Guttmann-Bauman and Karen Rubin were instrumental in articulating survey aims, participated in survey design and content, data analysis, the interpretation of findings and writing of the manuscript. Tyler Hamby analyzed the data and assisted with writing and editing. Michael Wood participated in data analysis and interpretation of findings and writing of the manuscript. Kent Reifschneider, Soumya Adhikari, and Paul Thornton aided in design of the survey questionnaire, interpretation of the results and edited the manuscript. All authors agreed with the final version of the manuscript.

\section{REFERENCES}

1. Dabelea D, Mayer-Davis EJ, Saydah S, et al. Prevalence of type 1 and type 2 diabetes among children and adolescents from 2001 to 2009. JAMA. 2014;311:1778-1786.

2. Maahs DM, West NA, Lawrence JM, Mayer-Davis EJ. Epidemiology of type 1 diabetes. Endocrinol Metab Clin North Am. 2010;39: 481-497.

3. Reinehr T. Type 2 diabetes mellitus in children and adolescents. World J Diabetes. 2013;4:270-281.

4. Pihoker C, Forsander G, Fantahun B, et al. The delivery of ambulatory diabetes care to children and adolescents with diabetes. Pediatric Diabetes. 2014;15(Suppl. 20):86-1015.

5. Miller KM, Foster NC, Beck RW, et al. Current state of type 1 diabetes treatment in the US: updated data from the T1D exchange clinic registry. Diabetes Care. 2015;38:971-978.

6. Markowitz JT, Volkening LK, Laffel LM. Care utilization in a pediatric diabetes clinic: cancellations, parental attendance, and mental health appointments. J Pediatr. 2014;164:1384-1389.

7. Rydon-Grange M. "What's psychology got to do with it?" Applying psychological theory to understanding failures in modern healthcare settings. J Med Ethics. 2015;41:880-884.

8. Ducat L, Rubenstein A, Philipson LH, Anderson BJ. A review of the mental health issues of diabetes conference. Diabetes Care. 2015;38: 333-338.

9. Lewin $A B$, Heidgerken $A D$, Geffken $G R$, et al. The relation between family factors and metabolic control: the role of diabetes adherence. $J$ Pediatr Psychol. 2006;31:174-183.

10. Schwartz DD, Cline VD, Axelrad ME, Anderson BJ. Feasibility, acceptability, and predictive validity of a psychosocial screening program for children and youth newly diagnosed with type 1 diabetes. Diabetes Care. 2011;34:326-331.

11. Winkley K, Landau S, Eisler I, Ismail K. Psychological interventions to improve glycaemic control in patients with type 1 diabetes: systematic review and meta-analysis of randomised controlled trials. BMJ. 2006;333:65-68.

12. Berwick DM, Nolan TW, Whittington J. The triple aim: care, health and cost. Health Aff (Millwood). 2008;27:759-769.

13. Asarnow JR, Rozenman M, Wiblin J, Zeltzer L. Integrated medical-behavioral care compared with usual primary Care for Child and Adolescent Behavioral Health: a meta-analysis. JAMA Pediatr. 2015;169:929-937.

14. Jasinski CF, Rodriguez-Monguio R, Tonyushkina K, Allen H. Healthcare cost of type 1 diabetes mellitus in new-onset children in a hospital compared to an outpatient setting. BMC Pediatr. 2013;13:55.

15. Tonyushkina $\mathrm{KN}^{1}$, Visintainer PF, Jasinski CF, Wadzinski TL, Allen HF. Site of initial diabetes education does not affect metabolic outcomes in children with T1DM. Pediatr Diabetes. 2014;15:135-141.

16. Klingensmith GJ, Allen HF. The cost of providing care to the pediatric patient with diabetes. Endocrinologist. 2001;11:41-47.

17. Rubin K, Oliva J, Croes D. Improving outcomes and reimbursement: outpatient management of pediatric diabetes and the implications for chronic illness. In: Cohn KH, Hough DE, eds. The Business of Healthcare. Vol 3. Westport Connecticut: Praeger Perspectives; 2007: 157-175

18. Vigersky RA, Fitzner K, Levinson J. Barriers and potential solutions to providing optimal guideline-driven care to patiens with diabetes in the US. Diabetes Care. 2013;36:3843-3849.

19. Melzer SM, Richards GE, Covington ML. Reimbursement and costs of pediatric ambulatory diabetes care by using the resource-based relative value scale: is multidisciplinary care financially viable? Pediatr Diabetes. 2004;5:133-142.

20. Porter ME, Kaplan RS. How to pay for health care. Harv Bus Rev. July-August 2016 2016;88-100.

21. Wood Colleen L, Clements Scott A, Kim MF, Robert S, Thomas John F, Paul WR. Use of telemedicine to improve adherence to American Diabetes Association standards in pediatric type 1 diabetes. Diabetes Technol Ther. 2016;18:7-14.

22. Curtis-Tyler K, Arai L, Stephenson T, Roberts H. What makes for a good or bad pediatric diabetes service from the viewpoint of children, 
young people, carers and clinicians? A synthesis of qualitative findings. Arch Dis Child. 2015;100(9):826-833.

23. Agiostratidiou G, Anhalt H, Ball D, et al. Standardizing clinically meaningful outcome measures beyond HbA1c for type 1 diabetes: a consensus report of the American Association of Clinical Endocrinologists, the American Association of Diabetes Educators, the American Diabetes Association, the Endocrine Society, JDRF International, The Leona M. and Harry B. Helmsley Charitable Trust, the Pediatric Endocrine Society and the T1D Exchange. Diabetes Care. 2017;40(12):1622-1630.
How to cite this article: Guttmann-Bauman I, Thornton P, Adhikari S, et al. Pediatric endocrine society survey of diabetes practices in the United States: What is the current state? Pediatr Diabetes. 2018;19:859-865. https://doi.org/10.1111/ pedi.12677 